\title{
A Comparison of Mobile Vehicles for Data Collection and Wireless Charging
}

\author{
Yu Sheng, Weirong Liu, Yating Li, Ping Zhong ${ }^{*}$, and Guihua Duan \\ School of Computer Science and Engineering, Central South University, Changsha, 410083, China
}

\begin{abstract}
There are two ways to collect data and charge nodes based on mobile vehicles in wireless rechargeable sensor networks (WRSNs). One involves using single function vehicles, data collection vehicles (DCVs), and wireless charging vehicles (WCVs) to collect data and supply energy to sensors. The other involves using dual function vehicles, simultaneous data collection, and wireless charging vehicles (DC-WCVs) to gather data and supply energy to sensors. In order to compare and analyze the performance difference of these two ways, we classify and summarize related works in the field of mobile vehicles in sensor networks. Furthermore, we validate the network performance differences between the two ways through experimental verification and observe that the network lifetime and collected data amount are higher in networks using single function vehicles. The tour length of dual function vehicles is smaller in networks using dual function vehicles. Finally, we propose suitable vehicles to collect data and charge nodes for different optimization objectives.
\end{abstract}

Keywords: single function vehicles; dual function vehicles; wireless rechargeable sensor networks; network performance difference

(Submitted on June 11, 2019; Revised on July 12, 2019; Accepted on August 6, 2019)

(C) 2019 Totem Publisher, Inc. All rights reserved.

\section{Introduction}

Information acquisition, transmission, processing, and utilization are important components of informationization. Information acquisition is one of the important links of informationization [1]. With the rapid development of communication technology, wireless charging technology based on radio frequency, and energy collection technology, wireless rechargeable sensor networks (WRSNs) have been widely used to collect data in environment monitoring, information transmission, home automation, and traffic control [2]. It has become one of the important means of monitoring environments and acquiring information [3]. However, the sensor energy for communication comes from energy acquisition modules and energy storage modules. Efficient data collection is closely related to node battery energy, data transmission routing, distribution of nodes, link constraints, and base station location. That makes the data collection a key research issue in WRSNs [4-6].

Many scholars have conducted research recently to address the energy constraints. A few works have studied using wireless charging vehicles (WCVs) equipped with resonance coils to supply energy based on wireless energy transfer (WET) technology. WCVs not only reduce the energy dissipation, but also can better adapt to network topology changes [7-9]. For data collection, instead of deploying static sink nodes [10-13] or dynamic sink nodes [14-16] to collect data by multi-hop routing transmission, data collection vehicles (DCVs) are widely used to collect data. The combination of DCVs and multihop transmission not only balances the load of sensor nodes, but also reduces the energy consumption by relaying and forwarding data [17-19].

In addition to collecting data and charging node by DCVs and WCVs, dual function vehicles, simultaneous data collection, and wireless charging vehicles (DC-WCVs) are used to collect data and charge nodes in WRSNs [20-22]. In WRSNs, the utilization of both single and dual function mobile vehicles to collect data and supply energy prolongs the network lifetime, reduces the network consumption, and improves the network performance, while can achieve the

* Corresponding author.

E-mail address: ping.zhong@csu.edu.cn 
optimization objectives [23-25]. However, no work has proposed which data collection and energy supplement is more suitable for different optimization objectives.

In order to compare and analyze the differences of the above two ways of data collection and energy supplement, this paper divides the network scenarios into two categories. The network scenarios are shown in Figure 1. In scenario 1, DCV and WCV are deployed in the sensing area to collect data and supply energy to sensor nodes. DCV is responsible for collecting anchors' data, and WCV is responsible for supplying energy to sensors waiting to be charged. The two kinds of vehicles perform their own duties and do not interfere with each other. In scenario 2, a dual function vehicle DC-WCV is deployed in the sensing area to collect data and charge nodes. DC-WCV's service object can be either the anchors or the nodes waiting to be charged. DC-WCV can collect data and charge nodes simultaneously. The data collection and wireless charging can influence each other. In addition, DCV, WCV, and DC-WCV all use a one-to-one service mode. Under the condition of using the same network parameters and algorithms, this paper compares and analyses the network performance differences for the two scenarios.

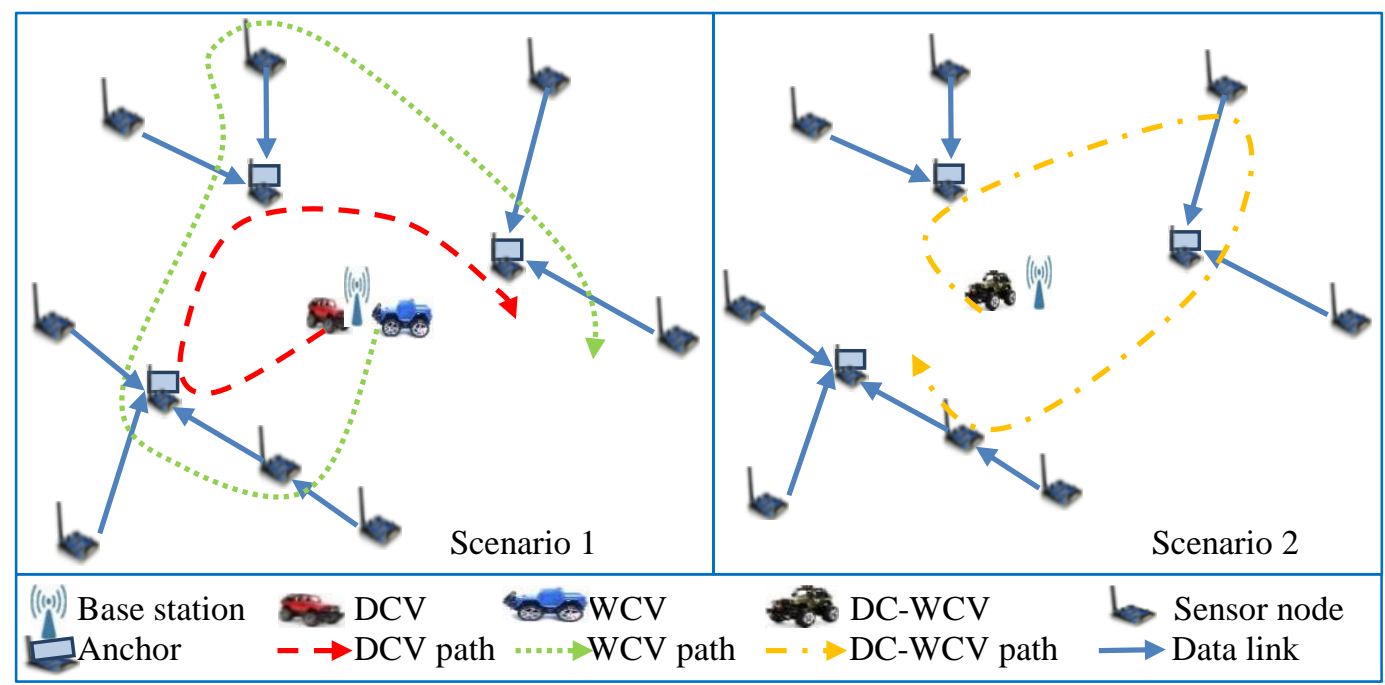

Figure 1. Network scenario diagram for scenario 1 with DCV and WCV, and scenario 2 with DC-WCV

\section{Scheduling Algorithm based on Mobile Vehicles in WRSN}

With the development of wireless energy transfer technology and wireless communication technology, several recent works have studied using mobile vehicles to charge nodes and collect data. This paper divides the network scenarios into two categories based on mobile vehicles' functions. Thus, there are two scheduling algorithms of data collection and energy supplement. This section consists of two subsections: scheduling algorithms based on single function vehicles and scheduling algorithms based on dual function vehicles.

\subsection{Scheduling Algorithms based on Single Function Vehicles}

Single function vehicle DCV completes the whole data collection process in three steps, which are network partition, anchor selection, and DCV path construction. In addition, single function vehicle WCV constructs a charging queue and generates a WCV moving path to finish the energy supplement process.

For sensor networks only using DCVs, Ghaleb et al. [26] finished the data collection process using one DCV. Sensory data was transmitted to the base station by multi-hop transmission and mobile vehicle transmission. In order to solve the data collection problem, the authors divided the whole network into two regions, the inner region and the outer region, using a minimum spanning tree. In the inner region, the nodes transmitted data to the base station through a multi-hop relay mode. In the outer region, some sensors were selected as anchors based on the minimum spanning tree. The DCV path was the shortest anchor path constructed by the TSP algorithm. Finally, DCV collected data by moving to each anchor. In [27], DCV was used to collect sensory data. Sensors generated data at a fixed rate. Some sensors were selected as anchors to collect data within their $\mathrm{k}$ hop coverage. Then, DCV started from the base station and visited anchors at a fixed speed. Eventually, it returned to the base station and transmitted collected data to the base station. An energy-aware path construction was proposed to complete the data collection process. The algorithm could prolong the network lifetime by reducing DCV's moving path length so that the vehicle can visit as many anchors as possible. 
Due to the limited speed of mobile vehicles in large scale networks, sensory data cannot be collected in time. In order to solve this problem, a time adaptive schedule algorithm (TASA) was proposed in [28] and uses several DCVs to finish the data collection process. TASA consists of three steps: network partition and routing design, anchor selection algorithm, and time schedule algorithm. It can transmit collected data to cloud anywhere in the network. The design of multiple vehicles and cloud can reduce the data collection time and ensure data delay constraints.

For sensor networks only using WCVs, one WCV was used to supply energy wirelessly in [29]. Due to underestimating the impact of time and space constraints in the charging process, the traditional schedule strategy in on-demand WRSN has a lower charging request throughput and success rate. Therefore, a TemporAl \& Distantial Priority charging scheduling algorithm (TADP) was proposed [29], which considers the distance between sensors and WCV and the waiting time of the charging request. WCV can achieve a balance between the sensors' residual time and moving path, which can help WCV select the optimal node to be charged. Finally, WCV moves to the node and replenishes its energy.

In the sensor networks using DCVs and WCVs, multiple DCVs and WCVs were used to collect data and charge nodes to maximize the collected data amount in [30]. Because of the limited energy of vehicles, a twice-partition algorithm based on center points (TP-CP) was proposed to meet the energy and charging requirements of all sensors. In addition, an anchor selection algorithm based on the trade-off between neighbor amount and residual energy, named AS-NAE, was proposed to collect the zonal data. In [30], the paper aimed to maximize the network utility considering energy constraints of sensors and vehicles, link bandwidth constraints, and data flow constraints. The network utility function was defined as a second order derivable non-convex function for collected data amounts. This paper first transformed the network collection optimization problem into its dual problem and proposed a distributed algorithm to solve the dual problem. In [31], one DCV and multiple WCVs were used to collect data and replenish energy. In order to reduce data delays, some circles of the same size were employed to cover the whole sensor network. The centers of these circles were regarded as anchors to collect data in its circular area. Then, DCV constructed the shortest path of anchors through the TSP algorithm. In terms of charging strategy, taking energy constraints and delay constraints into consideration, the authors proposed an adaptive network partition algorithm and deployed a WCV in each area to supply energy through the greedy algorithm.

Although most scenarios based on single function vehicles use three steps in the data collection process, the emphasis of each algorithm is different. Table 1 provides a summary of single function vehicle scheduling algorithms. "-" means the relevant work has not been mentioned in this paper. Table 1 lists the types and numbers of vehicles, network model, and main measure indexes in each step.

Table 1. Summary of single function vehicle scheduling algorithms

\begin{tabular}{|c|c|c|c|c|c|c|c|}
\hline Literature & Network model & DCV & WCV & Network partition & Anchor selection & DCV path & Charging queue \\
\hline $\begin{array}{c}\text { Ghaleb et al., } \\
2016\end{array}$ & WSN & One & - & $\begin{array}{l}\text { Based on minimum } \\
\text { spanning tree }\end{array}$ & $\begin{array}{l}\text { Based on minimum } \\
\text { spanning tree }\end{array}$ & $\begin{array}{l}\text { Based on } \\
\text { TSP } \\
\text { algorithm } \\
\end{array}$ & - \\
\hline $\begin{array}{l}\text { Wen et al., } \\
2018\end{array}$ & WSN & One & - & - & $\begin{array}{c}\text { Based on minimum } \\
\text { spanning tree and } \\
\text { sensor load }\end{array}$ & $\begin{array}{l}\text { Based on } \\
\text { TSP } \\
\text { algorithm }\end{array}$ & - \\
\hline $\begin{array}{l}\text { Wang et al., } \\
2017\end{array}$ & WSN & One & - & $\begin{array}{c}\text { Based on center } \\
\text { angle }\end{array}$ & $\begin{array}{c}\text { Minimum spanning } \\
\text { tree and node } \\
\text { sociality }\end{array}$ & $\begin{array}{l}\text { Based on } \\
\text { TSP } \\
\text { algorithm }\end{array}$ & 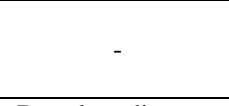 \\
\hline $\begin{array}{l}\text { Lin et al., } \\
2016\end{array}$ & WRSN & - & One & - & ( & - & $\begin{array}{c}\text { Based on distance } \\
\text { and waiting time of } \\
\text { charging request }\end{array}$ \\
\hline $\begin{array}{l}\text { Zhong et al., } \\
2017\end{array}$ & WRSN & Multiple & Multiple & $\begin{array}{c}\text { Based on routing } \\
\text { and distance }\end{array}$ & $\begin{array}{c}\text { Based on neighbor } \\
\text { amount and residual } \\
\text { energy }\end{array}$ & $\begin{array}{l}\text { Based on } \\
\text { TSP } \\
\text { algorithm } \\
\end{array}$ & $\begin{array}{c}\text { Based on waiting } \\
\text { time of charging } \\
\text { request }\end{array}$ \\
\hline $\begin{array}{l}\text { Wang et al., } \\
2016\end{array}$ & WRSN & One & Multiple & Based on distance & $\begin{array}{c}\text { Based on center of a } \\
\text { circle }\end{array}$ & $\begin{array}{l}\text { Based on } \\
\text { TSP } \\
\text { algorithm } \\
\end{array}$ & $\begin{array}{l}\text { Based on greedy } \\
\text { algorithm }\end{array}$ \\
\hline
\end{tabular}

\subsection{Scheduling Algorithms based on Dual Function Vehicles}

When dispatching dual function vehicle DC-WCV to serve sensors in a network, there are generally two steps, anchor selection and DC-WCV path construction.

In [32], one DC-WCV was deployed to collect data and supply energy. A new framework was proposed for WRSN. It divides the DC-WCV path into two types, the charging path and the data collection path. The two paths perform its function without interference, and they are alternate. DC-WCV can collect data from anchors while supplying energy to nodes, 
which prolongs the sensors' lifetime and reduces the data collection delay [33]. In addition, the sensors with the least energy are selected as anchors. In [34], multiple DC-WCVs are responsible for data collection and wireless charging. Each sensor can be serviced by designing DC-WCVs paths. The paths vary with time, which effectively reduces data delays and communication energy consumption. In [35], multiple DC-WCVs were deployed to collect data and supply energy. Taking charging distance, energy, and buffer constraints into consideration, this paper aims at maximizing the network lifetime by scheduling the least number of DC-WCVs. Hence, the periodic energy replenishment and data collection problem is proposed. DC-WCVs move to each anchor to collect data and replenish energy for sensors in the DC-WCVs' coverage area. There are three anchor selection algorithms: the grid-based algorithm (GBA), the dominating-set-based algorithm (DSBA), and the circle-intersection-based algorithm (CIBA). Finally, each feasible path is assigned one DC-WCV to achieve the optimization goal.

Table 2 provides a summary of dual function vehicle scheduling algorithms. "-" means the relevant work has not been mentioned in the paper. Table 2 lists the network model, number of vehicles, service mode, anchor selection, and DC-WCV path construction.

Table 2. Summary of dual function vehicle scheduling algorithms

\begin{tabular}{|c|c|c|c|c|c|}
\hline Literature & Network model & DC-WCV & Service mode & Anchor selection & DC-WCV path \\
\hline Zhao et al., 2014 & WRSN & One & One-to-one & $\begin{array}{c}\text { Based on residual energy } \\
\text { within } k \text { hops }\end{array}$ & Based on TSP algorithm \\
\hline Guo et al., 2014 & WRSN & One & One-to-one & Based on residual energy & Based on TSP algorithm \\
\hline Farris et al., 2015 & WRSN & Multiple & One-to-one & - & Based on TSP algorithm \\
\hline Liu et al., 2017 & WRSN & Multiple & One-to-many & GBA, DSBA, CIBA & Based on TSP algorithm \\
\hline
\end{tabular}

\section{Comparison of Network Performance in Two Scenarios}

In order to further analyse and compare the network performance under the two network scenarios, this paper considers a rechargeable sensor network consisting of 100 sensor nodes, four DCVs, four WCVs, and four DC-WCVs. The base station is located at the center of the whole sensor network. The network topology is shown in Figure 2. Sensors are uniformly distributed in a sensing area of $100 \mathrm{~m} \times 100 \mathrm{~m}$. The red pentagon represents the location of the base station. The number in Figure 2 represents the indices of sensors. The connections in the network indicate that two nodes can communicate with each other directly. This paper evaluates the performance of the two scenarios in terms of network lifetime, energy consumption of sensor nodes, tour lengths of mobile vehicles, and collected data amount. The network lifetime is the period from the beginning of network operation to the death of the first sensor node. Once the energy of sensors is exhausted, death sensors will not be able to relay or transmit sensory data to mobile vehicles. This will lead to sensory data loss. A longer network lifetime allows for the collection of more sensory data. The energy consumption of sensor nodes is the difference value between the initial energy and the current energy of sensor nodes in a time period. A smaller difference value means more residual energy of sensor nodes in the network. The tour lengths of vehicles are the single function vehicles and dual function vehicles. Since the vehicles' energy capacity is limited and the mobile energy consumption per unit time is greater than communication energy, the tour length is an important factor affecting data collection and energy supplement. The numerical experiments are conducted by MATLAB.

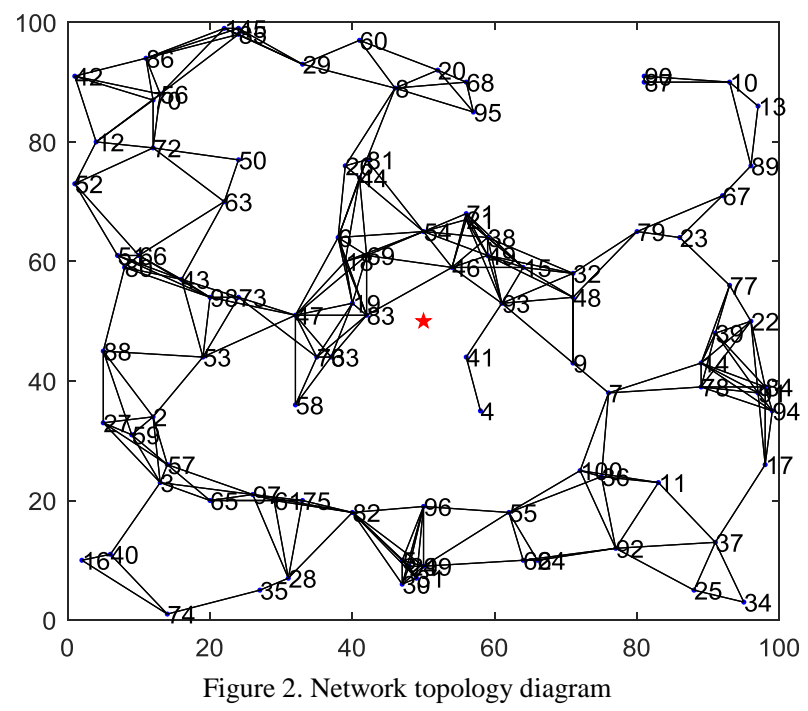


All sensor nodes in the network have the same hardware configuration. The data generation rate obeys the Poisson distribution with a mean value of $\lambda=4 \mathrm{~B} / \mathrm{s}$ based on [31]. The energy model was used in [36]. Considering the temperature monitoring system in [36], network parameter settings are shown in Table 3. In order to evaluate the network performance difference between the two scenarios, the network parameter settings are constant. The network partition algorithm TP-CP [30] and anchor selection algorithm AS-NAE [30] are used in the two scenarios. Based on the vehicles' path planning algorithm in Table 1 and Table 2, this paper uses the TSP algorithm to construct the data collection path. This not only ensures that each anchor is visited once per round, but also shortens the tour length of vehicles. To solve the energy replenishment problem, recent works have designed mobile vehicles charging algorithms based on sensor residual energy, mobile energy consumption, and waiting time of charging requests. Furthermore, the TADP [29] algorithm considers the distance between sensors and WCV and the waiting time of charging request. The service mode in [29] is similar to DCV and WCV, which is one-to-one service. Therefore, the TADP algorithm is selected to determine the order of charging sensors. In networks using single function vehicles, DCV constructs the shortest path of anchors using the TSP algorithm. WCV chooses the node from the charging queue using the TADP algorithm. In networks using dual function vehicles, DCWCV firstly constructs the shortest path of anchors by the TSP algorithm. Then, the node to be charged from the charging queue is chosen by TADP. It is inserted into the DC-WCV path on the energy constraint and buffer constraint. Finally, a DC-WCV path composed of anchors and rechargeable nodes is rebuilt by the TSP algorithm.

Table 3. Parameter settings

\begin{tabular}{|c|c|}
\hline Parameter & Value \\
\hline Data transmission rate from sensor to vehicle & $50 \mathrm{~B} / \mathrm{s}$ \\
\hline Energy transmission efficiency from vehicle to sensor & $40 \%$ \\
\hline Moving velocity of the vehicle & $3 \sim 9 \mathrm{~m} / \mathrm{s}$ \\
\hline Sensor battery capacity & $50 \mathrm{~J}$ \\
\hline Vehicle battery capacity & $50 \mathrm{KJ}$ \\
\hline Sensor buffer size & $10 \mathrm{M}$ \\
\hline Delay constrain & $800 \mathrm{~s}$ \\
\hline Energy consumed for moving & $8.27 \mathrm{~m} / \mathrm{s}$ \\
\hline Energy transmission rate from vehicle to sensor & $1 \mathrm{~J} / \mathrm{s}$ \\
\hline One hop transmission delay & $1 \mathrm{~s}$ \\
\hline
\end{tabular}

Figure 3 shows the network lifetime change as the vehicle speed increases. The network lifetime is the time when first dead sensor appears. It can be seen from Figure 3 that as the vehicle speed increases, the dead sensor appears later. This is because the data generation rate and the anchor selection algorithm are the same in the two scenarios. When the moving speed increases, vehicles can quickly reach the node to be served. It can supply energy to more sensors in the same period and prolong the network lifetime. Therefore, the network lifetime can be improved by raising vehicles' speed. In addition, we can see that the network lifetime using single function vehicles is greater than thas ung dual function vehicles when the speed is constant. This is because DC-WCV needs to charge normal nodes aside from collecting data from anchors. When DC-WCV is powered for non-anchored nodes, it does not collect data from charging nodes. Due to the limited capacity of vehicle batteries, it is necessary to give up on supplementing energy for some nodes to be charged. However, in networks using single function vehicles, DCV is responsible for collecting data while WCV is only responsible for charging nodes. WCV has smaller tasks than DC-WCV, which makes WCV charge more nodes. Hence, the network lifetime is longer when using single function vehicles.

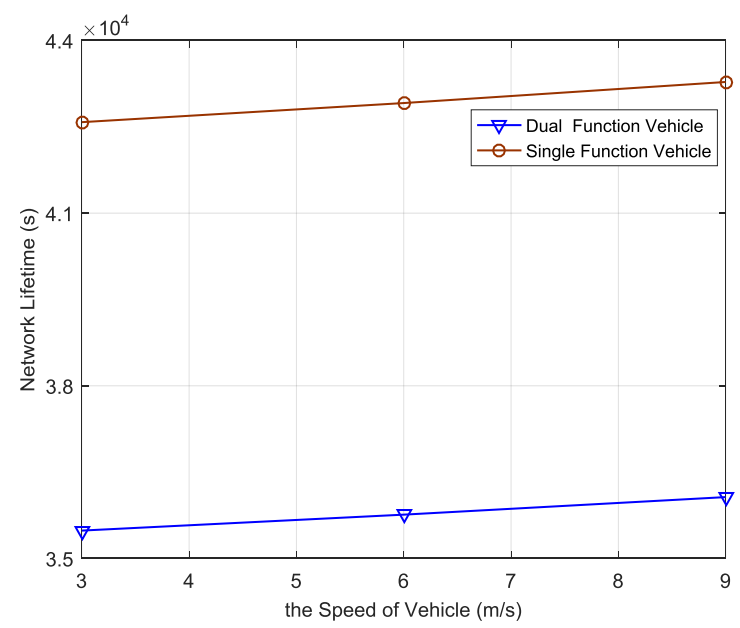

Figure 3. Comparison of network lifetime in two scenarios 
Figure 4 shows the trend of total energy consumption of sensors in the two scenarios. The value is obtained by subtracting the sum of the initial sensor energy and the current time sensor energy. It can be seen from Figure 4 that the total energy consumption of sensors increases gradually. Sensors generate sensory data all the time, and the energy consumption increases with time. In addition, the energy consumption in the two scenarios is the same within 0 to 6 hours. The energy consumption using single function vehicles is less than that using dual function vehicles within 6 to 10 hours. Because there is no charging node in the network and the data transmission links are the same, the node energy consumption is equal within 0 to 6 hours. However, the existence of charging nodes makes the load of DC-WCV increase within 6 to 10 hours. When there are more sensors to be charged besides anchors, WCV can serve more sensors than DC-WCV, and the network consumption decreases accordingly. Thus, energy consumption is lower when using single function vehicles.

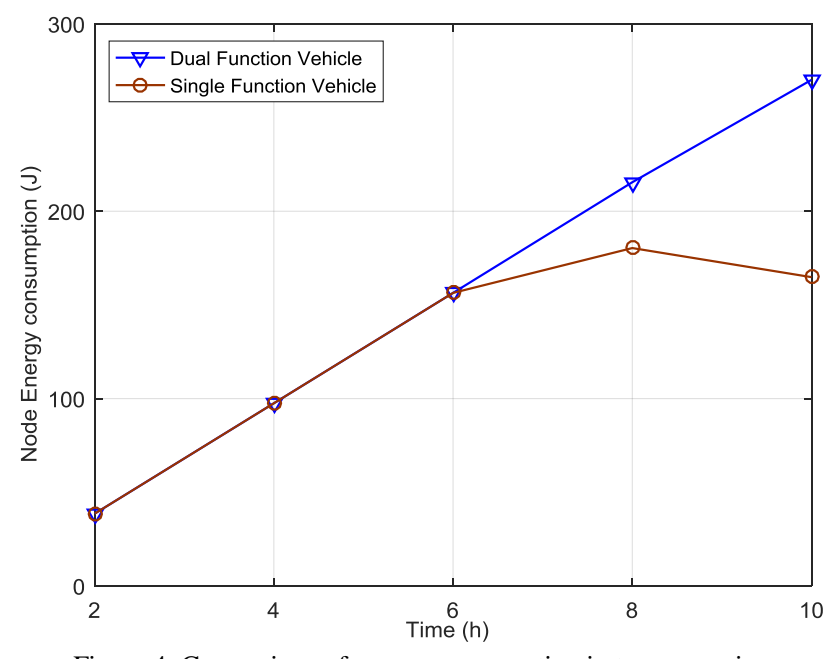

Figure 4. Comparison of energy consumption in two scenarios

Figure 5 shows the comparison of tour lengths of mobile vehicles in the two scenarios. In the single function vehicle scenario, the tour length is the sum of the DCV moving path and the WCV moving path. In the dual function vehicle scenario, the tour length is the DC-WCV moving path. As shown in Figure 5, the tour lengths of vehicles gradually increase. This is because vehicles are moving, transmitting, and receiving data all the time. The tour lengths are the same in the two scenarios within 0 to 6 hours. The tour length of DC-WCV is lower than the single function vehicle of DCV and WCV within 6 to 10 hours. Charging nodes do not exist within the network, which makes the service queue of DCV and DCWCV the same. The tour length of WCV is equal to zero. Finally, the tour lengths of the two scenarios are the same within 0 to 6 hours. Within 6 to 10 hours, there are more nodes to be charged. DC-WCV needs to insert a charging node into its service queue, while WCV needs to start from the base station to supply energy to sensors. This makes the tour length of single function vehicles longer than that of dual function vehicles.

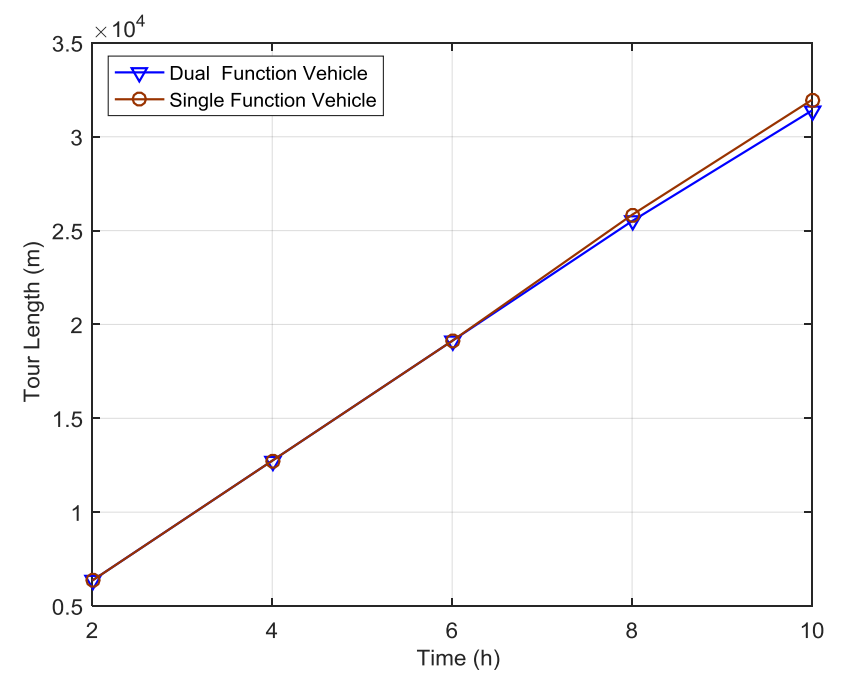

Figure 5. Comparison of tour length in two scenarios 
Figure 6 shows the collected data amount under the two scenarios. In Figure 6, the collected data amounts all increase. Moreover, the data amount collected by DCV is the same as that collected by DC-WCV within 0 to 6 hours. The data amount collected by DCV is higher than that collected by DC-WCV within 6 to 10 hours. This phenomenon reflects the effect of charging nodes. When a network has no charging nodes, the service queue composed by anchors of DC-WCV is the same as that composed by anchors of DCV. Anchors and data transmission links are similar in the two scenarios. Thus, the data amounts collected by DCV and DC-WCV are equal from 0 to 6 hours. When a charging node in the service queue appears in DC-WCV, the anchors, data transmission links, and service queue for DC-WCV and WCV are changed. The load of DC-WCV is more than that of DCV or WCV. Hence, the data amount collected by single function vehicles is higher than that collected by dual function vehicles.

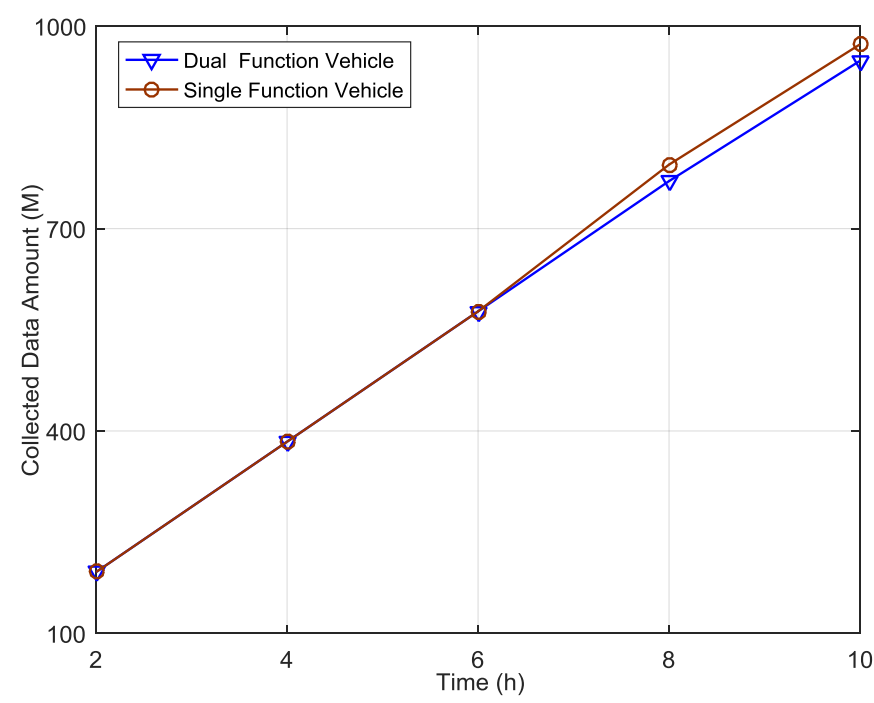

Figure 6. Comparison of collected data amount in two scenarios

\section{Conclusions}

This paper divides the network scenario into two categories according to mobile vehicles. For the two scenarios, this paper summarizes the network type, vehicle function, data collection algorithms, and wireless charging algorithms. Finally, on the conditions of using the same network parameter settings, the network partition algorithm, and the anchor selection algorithm in the two scenarios, we compare the network performance including the network lifetime, energy consumption, vehicle tour length, and collected data amount. Experiments show that the network lifetime and energy consumption in the scenario using single function vehicles is better than those in the scenario using dual function vehicles. The tour length of single function vehicles is higher than that of dual function vehicles, which makes the energy consumption higher in the scenario using single function vehicles.

In this paper, we assume that DC-WCV in a partition is one. One DCV and one WCV are deployed in a partition. However, the vehicle parameter settings are the same, which makes the collected data amount and network lifetime high in the network using single function vehicles. In our future work, we will future consider deploying two DC-WCVs rather than one DC-WCV in a partition and compare the performance between the two scenarios.

\section{Acknowledgements}

This work was supported by the National Natural Science Foundation of Hunan Province, China (No. 2018JJ3692), the National Natural Science Foundation of China (No. 61672539), and the Fundamental Research Funds for the Central Universities of Central South University (No. 2018zzts599).

\section{References}

1. I. F. Akyildiz, W. Su, Y. Sankarasubramaniam, and E. Cayirci, "Wireless Sensor Networks: A Survey," Computer Networks, Vol. 38, No. 4, pp. 393-422, March 2002

2. Y. Yang and C. Wang, "Wireless Rechargeable Sensor Networks," Springer International Publishing, West Berlin, Germany, 2015

3. J. Yick, B. Mukherjee, and D. Ghosal, "Wireless Sensor Network Survey," Computer Networks, Vol. 52, No. 12, pp. 2292-2330, 
August 2008

4. M. Dong, K. Ota, A. Liu, and M. Guo, "Joint Optimization of Lifetime and Transport Delay under Reliability Constraint Wireless Sensor Networks," IEEE Transactions on Parallel \& Distributed Systems, Vol. 27, No. 1, pp. 225-236, January 2016

5. J. Ren, Y. Zhang, K. Zhang, A. Liu, J. Chen, and X. Shen, "Lifetime and Energy Hole Evolution Analysis in Data-Gathering Wireless Sensor Networks," IEEE Transactions on Industrial Informatics, Vol. 12, No. 2, pp. 788-800, April 2016

6. L. Jiang, A. Liu, Y. Hu, and Z. Chen, "Lifetime Maximization through Dynamic Ring-based Routing Scheme for Correlated Data Collecting in WSNs," Computers \& Electrical Engineering, Vol. 41, pp. 191-215, January 2015

7. C. Wang, J. Li, Y. Yang, and F. Ye, "A Hybrid Framework Combining Solar Energy Harvesting and Wireless Charging for Wireless Sensor Networks," in Proceedings of the 35th Annual IEEE International Conference on Computer Communications, pp. 1-9, San Francisco, CA, USA, April 2016

8. Y. Shu, H. Yousefi, P. Cheng, J. Chen, Y. Gu, T. He, et al., "Near-Optimal Velocity Control for Mobile Charging in Wireless Rechargeable Sensor Networks," IEEE Transactions on Mobile Computing, Vol. 15, No. 7, pp. 1699-1713, July 2016

9. X. Lu, P. Wang, D. Niyato, D. I. Kim, and Z. Han, "Wireless Networks with RF Energy Harvesting: A Contemporary Survey," IEEE Communications Surveys \& Tutorials, Vol. 17, No. 2, pp. 757-789, 2015

10. G. Abdul-Salaam, A. H. Abdullah, M. H. Anisi, A. Gani, and A. Alelaiwi, "A Comparative Analysis of Energy Conservation Approaches in Hybrid Wireless Sensor Networks Data Collection Protocols," Telecommunications Systems, Vol. 61, No. 1, pp. 159-179, January 2016

11. K. Narendra, V. Varun, and G. H. Raghunandan, "A Comparative Analysis of Energy-Efficient Routing Protocols in Wireless Sensor Networks," Emerging Research in Electronics, Computer Science and Technology, pp. 399-405, 2014

12. F. Yuan, Y. Zhan, and Y. Wang, "Data Density Correlation Degree Clustering Method for Data Aggregation in WSN," IEEE Sensors Journal, Vol. 14, No. 4, pp. 1089-1098, April 2014

13. P. Du, Q. Yang, Z. Shen, and K. S. Kwak, "Quality of Information Maximization in Lifetime-Constrained Wireless Sensor Networks,” IEEE Sensors Journal, Vol. 16, No. 19, pp. 7278-7286, October 2016

14. J. Wang, B. Li, F. Xia, C. Kim, and J. Kim, "An Energy Efficient Distance-Aware Routing Algorithm with Multiple Mobile Sinks for Wireless Sensor Networks," Sensors, Vol. 14, No. 8, pp. 15163-15181, August 2014

15. M. Zhong and C. G. Cassandras, "Distributed Coverage Control and Data Collection with Mobile Sensor Networks," IEEE Transactions on Automatic Control, Vol. 56, No. 10, pp. 2445-2455, January 2011

16. Y. Zhang, S. He, and J. Chen, "Near Optimal Data Gathering in Rechargeable Sensor Networks with a Mobile Sink," IEEE Transactions on Mobile Computing, Vol. 16, No. 6, pp. 1718-1729, January 2016

17. L. He, J. Pan, and J. Xu, "A Progressive Approach to Reducing Data Collection Latency in Wireless Sensor Networks with Mobile Elements," IEEE Transactions on Mobile Computing, Vol. 12, No. 7, July 2013

18. M. Zhao and Y. Yang, "Bounded Relay Hop Mobile Data Gathering in Wireless Sensor Networks," IEEE Transactions on Computers, Vol. 61, No. 2, pp. 265-277, February 2011

19. X. Zhang, H. Dai, L. Xu, and G. Chen, "Mobility-Assisted Data Gathering Strategies in WSNs," Journal of Software, Vol. 24, No. 2, pp. 198-214, 2014

20. S. Gao, H. Zhang, and S. K. Das, "Efficient Data Collection in Wireless Sensor Networks with Path-Constrained Mobile Sinks," IEEE Transactions on Mobile Computing, Vol. 10, No. 4, pp. 592-608, April 2011

21. X. Ren, W. Liang, and W. Xu, "Use of a Mobile Sink for Maximizing Data Collection in Energy Harvesting Sensor Networks," in Proceedings of International Conference on Parallel Processing, pp. 439-448, April 2013

22. C. Wang, J. Li, F. Ye, and Y. Yang, "Recharging Schedules for Wireless Sensor Networks with Vehicle Movement Costs and Capacity Constraints," in Proceedings of the 11th IEEE International Conference on Sensing, Communication, and Networking, pp. 468-476, Singapore, June 2014

23. M. Ma and Y. Yang, "SenCar: An Energy-Efficient Data Gathering Mechanism for Large-Scale Multihop Sensor Networks," IEEE Transactions on Parallel \& Distributed Systems, Vol. 18, No. 10, pp. 1476-1488, October 2007

24. M. Zhao, D. Gong, and Y. Yang, "Network Cost Minimization for Mobile Data Gathering in Wireless Sensor Networks," IEEE Transactions on Communications, Vol. 63, No. 11, pp. 4418-4432, November 2015

25. C. Wang, S. Guo, and Y. Yang, "An Optimization Framework for Mobile Data Collection in Energy-Harvesting Wireless Sensor Networks," IEEE Transactions on Mobile Computing, Vol. 15, No. 12, pp. 2969-2986, December 2016

26. M. Ghaleb, S. Subramaniam, M. Othman, and Z. Zukarnain, "An Efficient Hybrid Data Gathering Algorithm based on MultiHop and Mobile Element in WSNs," Turkish Journal of Electrical Engineering \& Computer Sciences, Vol. 25, No. 1, pp. 605621,2017

27. W. Wen, S. Zhao, C. Shang, and C. Chang, "EAPC: Energy-Aware Path Construction for Data Collection using Mobile Sink in Wireless Sensor Networks," IEEE Sensors Journal, Vol. 18, No. 2, pp. 890-901, January 2018

28. T. Wang, Y. Li, G. Wang, and J. Gao, "Sustainable and Efficient Data Collection from WSNs to Cloud," IEEE Transactions on Sustainable Computing, Vol. 4, No. 2, pp. 1-12, March 2017

29. C. Lin, Z. Wang, D. Han, Y. Wu, C. Yu, and G. Wu, "TADP: Enabling Temporal and Distantial Priority Scheduling for onDemand Charging Architecture in Wireless Rechargeable Sensor Networks," Journal of Systems Architecture, Vol. 70, pp. 2638, October 2016

30. Z. Ping, Y. Li, W. Liu, G. Duan, and Y. Chen, "Joint Mobile Data Collection and Wireless Energy Transfer in Wireless Rechargeable Sensor Networks," Sensors, Vol. 17, No. 8, pp. 1881, August 2017

31. C. Wang, J. Li, F. Ye, and Y. Yuan, "A Mobile Data Gathering Framework for Wireless Rechargeable Sensor Networks with Vehicle Movement Costs and Capacity Constraints," IEEE Transactions on Computers, Vol. 65, No. 8, pp. 2411-2427, August 2016

32. M. Zhao, J. Li, and Y. Yang, “A Framework of Joint Mobile Energy Replenishment and Data Gathering in Wireless 
Rechargeable Sensor Networks," IEEE Transactions on Mobile Computing, Vol. 13, No. 12, pp. 2689-2705, December 2014

33. S. Guo, C. Wang, and Y. Yang, "Joint Mobile Data Gathering and Energy Provisioning in Wireless Rechargeable Sensor Networks," IEEE Transactions on Mobile Computing, Vol. 13, No. 12, pp. 2836-2852, December 2014

34. I. Farris, L. Militano, A. Iera, A. Molinaro, and S. C. Spinella, "Tag-based Cooperative Data Gathering and Energy Recharging in Wide Area RFID Sensor Networks," Ad Hoc Networks, Vol. 36, No. P1, pp. 214-228, July 2015

35. B. H. Liu, N. T. Nguyen, V. T. Pham, and Y. Lin, "Novel Methods for Energy Charging and Data Collection in Wireless Rechargeable Sensor Networks," International Journal of Communication Systems, Vol. 30, No. 5, pp. 1-11, March 2017

36. S. Li, L. Fu, S. He, and Y. Sun, "Near-Optimal Co-Deployment of Chargers and Sink Stations in Rechargeable Sensor Networks," ACM Transactions on Embedded Computing Systems, Vol. 17, No. 1, pp. 1-10, 2017

Yu Sheng received his Ph.D. degree in computer science and Technology from Central South University, China. He is currently an associate professor in the school of computer science and engineering, Central South University. His research interests are natural language processing, virtualization and embedded systems.

Weirong Liu received his Ph.D. from the Institute of Automation at the Chinese Academy of Sciences. He is currently an associate professor in the School of Computer Science and Engineering at Central South University. His research interests are cooperative control, nonlinear control, wireless sensor networks, and embedded systems.

Yating Li received her BEng degree in computer and information engineering from Henan University in 2016. She is currently working toward an MS degree in computer science and engineering at Central South University. Her research interests include wireless sensor networks, network optimization, wireless communication, and mobile computing.

Ping Zhong received her Ph.D. in communication and information systems from Xiamen University in 2011. She is currently an associate professor in the School of Computer Science and Engineering at Central South University. Her current research interests include network optimization, wireless communication, mobile computing, and computer networks.

Guihua Duan received her Ph.D. in computer science and technology from Central South University in 2008. She is currently an associate professor in the School of Computer Science and Engineering at Central South University. Her current research interests include bioinformatics, information security, and computer networks. 